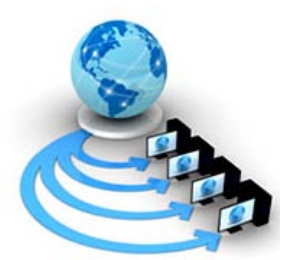

International Journal of Advanced Research in Computer Science

RESEARCH PAPER

\author{
Available Online at www.ijarcs.info
}

\title{
PERFORMANCE EVALUATION OF MLP AND RBF CLASSIFIERS FOR HANDWRITTEN CHARACTER RECOGNITION USING HYBRID FEATURES
}

\author{
Amit Choudhary \\ Department of Computer Science \\ Maharaja Surajmal Institute \\ New Delhi, India
}

\author{
Vinod Kumar \\ Department of Computer Science and Engineering \\ Delhi Technological University \\ Delhi, India
}

\begin{abstract}
For evaluating the accuracy and capability of MLP (Multi Layer Perceptron) and RBF (Radial Basis Function) classifier algorithms; projection profile features for the character images are extracted and are merged with the binarization features after pre-processing every character image, and the combined features thus obtained are used to train both the classifiers (MLP and RBF Network) selected for performing character recognition task. Simulation studies are examined extensively and the proposed hybrid features are found to deliver the recognition accuracy of $93.46 \%$ when used with RBF Network as a classifier which is better than the $88.76 \%$ accuracy obtained when used with MLP Network as a classifier.
\end{abstract}

Keywords: OCR, MLP, RBF, Feature Extraction, Projection Profile Features, Hybrid Features

\section{INTRODUCTION}

In the domain of off-line handwriting recognition, an (ANN) Artificial Neural Network became very popular in the late 70s and emerged as fast and most reliable classifier tool resulting in excellent recognition accuracy. The accuracy of the OCR System in recognizing the off-line handwritten character mainly depends on the selection of feature extraction technique and the classification algorithm employed. The motive behind this work is to compare the handwritten character recognition accuracy of the Multi-layered Perceptron (MLP) and Radial Basis Function Neural Network (RBFNN) classifiers trained with Hybrid Features (Fusion of Binarization and Projection Profile) extracted from the input character samples [1].

Both Multi-layered Perceptron (MLP) and Radial Basis Function (RBF) networks are basically non-linear layered feed forward networks. These networks work as universal approximators. RBF Neural Network is also a type of MLP but with Radial Basis as the activation function in the hidden layer neurons. It is therefore not surprising to find that there always exists an RBF network capable of accurately mimicking a specified MLP or vice versa [1, 2].

MLP may require a smaller number of parameters than the RBF network for the same degree of accuracy. But RBF Network is capable of implementing arbitrary non-linear transformations of the input space as illustrated by the XOR problem, which cannot be solved by any linear perceptron but can be solved by the RBF Network. Hence, the choice of the classifier lies in the problem domain. For different types of pattern classification activities, one neural network as a classifier may be proved to be superior to the other. In this paper, the superiority of the two classifiers is judged for the task of handwritten character recognition.

\section{FEATURE EXTRACTION USING BINARIZATION TECHNIQUE}

Feature extraction is a process of studying and deriving useful information from the filtered input patterns. The derived information can be general features, which are evaluated to ease further processing. The selection of features is very important because there might be only one-two values, which are significant to recognize a particular character. The performance of the recognition system relies much on the quality of the features extracted as well as on the selected classifier itself $[2,3]$.

The network is trained with 50 samples of handwritten characters where each sample has 26 characters (a-z). In the proposed experiment, $1300 \quad(50 \times 26=1300)$ handwritten characters have been involved.

After preprocessing of the handwritten character images, the features are extracted by using binarization technique for each character image from the local database of handwritten characters. As all the handwritten character images are resized to $15 \times 12$ (in order to make all the character images of uniform size), the feature vector of a single character is a column vector of size $180 \times 1$.

The binary image of character 'c', the resized image, Matrix representation of character ' $c$ ' and the feature vector of character 'c' are shown in Fig.1. As there are 26 characters, all the 26 feature vectors are combined to form a sample which is a binary matrix of size $180 \times 26$ as shown in Fig.2. 


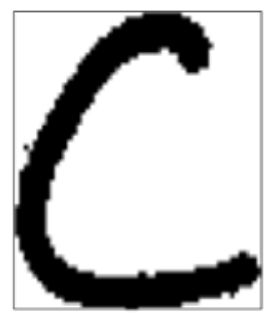

(a)

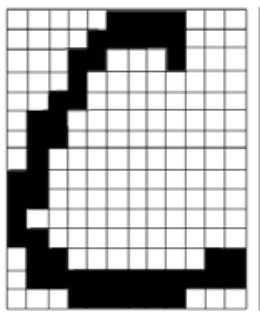

(b)

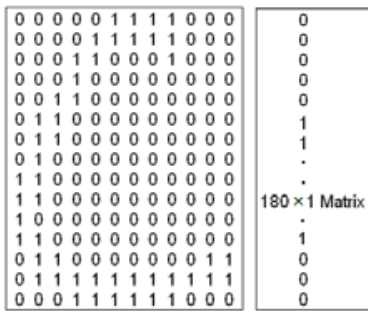

(c)

(d)

Figure 1. (a) Binary Image of Character 'c' (b) Resized Binary Image of Character 'c'; (c) Binary Matrix representation and (d) Reshaped Binary Matrix or Feature Vector of Character 'c'.

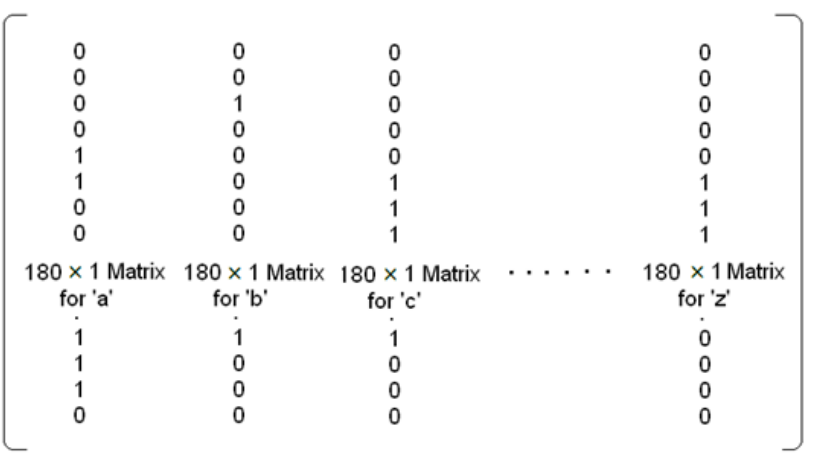

Figure 2. Matrix representation of Input Sample

The number of neurons in the input and output layers are fixed at 180 and 26 respectively. The 180 input neurons are equivalent to the input character's size as every character is resized into a binary matrix of size $15 \times 12$ and then reshaped to a matrix of size $180 \times 1$. The number of neurons in the output layer is 26 because there are 26 English alphabets. The number of neurons for the MLP classifier is kept 80 , by trial and error method, for optimal result.

\section{FEATURE EXTRACTION USING PROJECTION PROFILE}

Projection profile features in vertical, horizontal, left diagonal and right diagonal orientations to represent a character ' $c$ ' are shown in Fig.3. Projection features in the vertical direction is computed by tracing the character image column wise along the y-axis and counting the number of black pixels in each column. As the entire digit images are resized into $15 \times 12$ pixels, there are 12 columns and 15 rows [3].

Hence, the vertical projection profile will contain 12 values, each value representing the sum of number of all black pixels present in that particular column as shown in Fig.3(a). Similarly, for horizontal projection profile, character image is traced horizontally along the $\mathrm{x}$-axis. The row wise sum of number of black pixels present in each row will constitute the 15 values of horizontal projection profile as shown in Fig.3(b). Left diagonal projection profile is computed by traversing the character image along the left diagonal as shown in Fig.3(c). The black pixels are counted in left diagonal direction and the sum of the number of black pixels for each left diagonal line of traversing generates 26 values representing the left diagonal projection profile. In the same way, 26 values representing the right diagonal projection profile are obtained by adding the number of black pixels in each right diagonal line of traversing as shown in Fig.3 (d).
Projection profile values obtained in all the four orientations are combined to form a single feature vector $[2,4]$. The length of this feature vector is $79(12+15+26+26=79)$. As explained in Fig.3, a feature vector representing the character ' $c$ ' is obtained by concatenation of these four set of features and can be shown as:

$\{485544445122$

4531222122124116

00000365533111211222223210

$00012321111123232334354110\}$

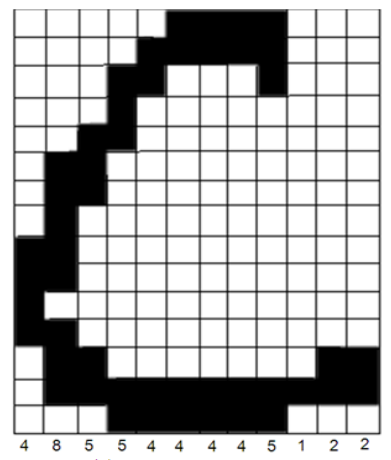

(a) Vertical Projection

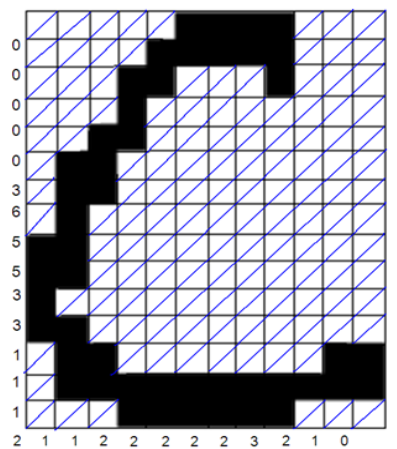

(c) Left Diagonal Projection

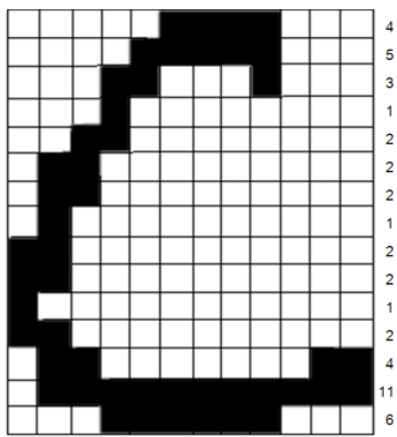

(b) Horizontal Projection

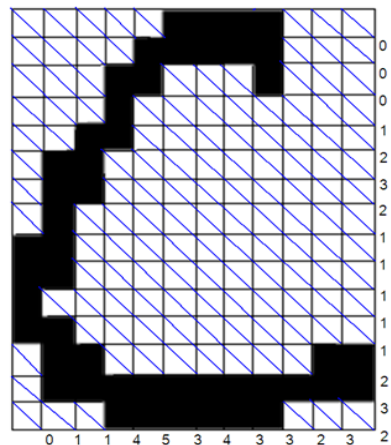

(d) Right Diagonal Projection
Figure 3. Projection Features of character 'c' in vertical, horizontal, left diagonal and right diagonal Orientations.

This projection profile feature vector of character ' $c$ ' can be represented in the form of a column matrix as shown in Fig.4.

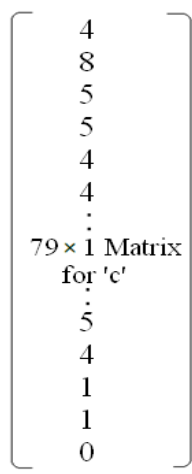

Figure 4. The Projection Feature Vector of Character 'c'.

The projection feature vectors of all the 26 characters (a-z) are created in the form of column matrix of size $79 \times 1$ each [3]. All these 26 feature vectors are combined to form a sample which is a binary matrix of size $79 \times 26$. 


$\left[\begin{array}{cccc}2 & 4 & 4 & 4 \\ 3 & 12 & 8 & 6 \\ 7 & 9 & 5 & 7 \\ 6 & 4 & 5 & 7 \\ 5 & 4 & 4 & 7 \\ 5 & 4 & 4 & 6 \\ \vdots & \vdots & 5 & \vdots \\ 79 \times \text { 1 Matrix } & 79 \times 1 \text { Matrix } & 79 \times \text { i Matrix } \ldots .79 \times \text { Matrix } \\ \text { for 'a' } & \text { for 'b' } & \text { for' 'c' } & \text { for' 'z' } \\ \vdots & \vdots & 5 & 4 \\ 6 & 4 & 4 & 5 \\ 2 & 4 & 1 & 5 \\ 1 & 2 & 1 & 3 \\ 0 & 0 & 0 & 1 \\ 0 & & & 5\end{array}\right]$

Figure 5. Matrix Representation of Input Training Sample of Size 79×26.

\section{SAMPLE PREPARATION USING HYBRID FEATURES EXTRACTION}

The feature vectors of each individual character using binarization and projection profile are combined. The process of combining involves the concatenation of the two feature vectors in such a way that the projection feature vector is appended at the end of the binarization feature vector. The combined feature vector is termed as "Hybrid Feature Vector" $[4,5]$. The process is repeated for all the characters in the character database. The hybrid feature vector for character ' $c$ ' obtained by fusion of binarization and projection feature vectors is shown in Fig.6.

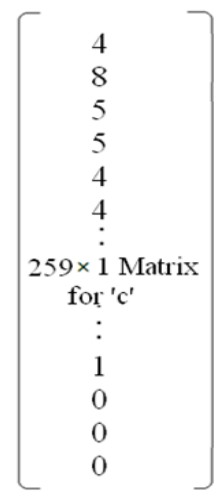

Figure 6. Hybrid Feature Vector of Character 'c'.

It can be observed that the length of the hybrid feature vector is 259 as the projection feature vector of length 79 is appended at the end of the binarization feature vector of length 180 [3].

The hybrid feature vectors of all the 26 characters (a-z) are created in the form of column matrix of size $259 \times 1$ each [8]. All these 26 feature vectors are combined to form a sample which is a binary matrix of size $259 \times 26$ as shown in Fig.7.
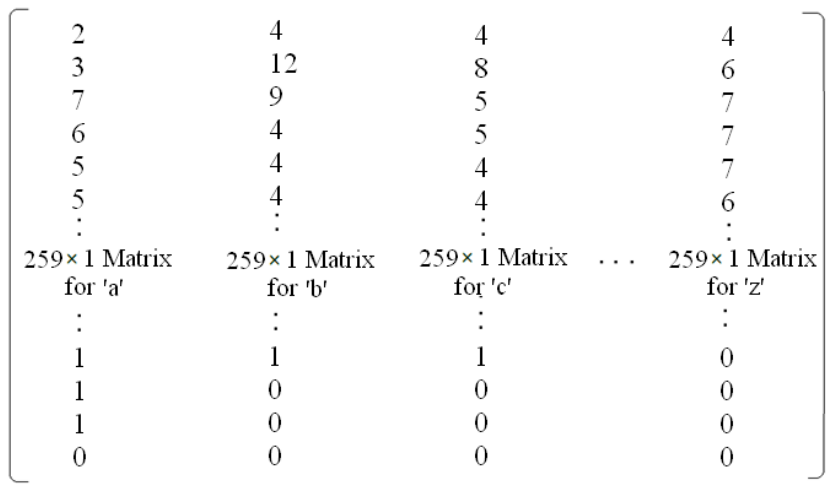

Figure 7. Input Sample Created by Fusion of Binarization and Projection Features.

\section{IMPLEMENTATION AND DISCUSSION OF RESULTS}

MLP neural network classifier has been trained with 50 sets of each character i.e. $1300(50 \times 26=1300)$ character image samples from the database has been involved in the training. Each character at the input will put a ' 1 ' at that neuron in the output layer in which the maximum trust is shown and rest neuron's result into ' 0 ' status. The output of both the network classifiers is binary matrix of size $26 \times 26$ each because there are 26 characters and every character has $26 \times 1$ output vector. The first $26 \times 1$ column stores the first character's recognition output; the following column will be for next character and so on for the whole sample [3].

During recognition, the confusion among the different characters is explained in Fig.8. Character 'a' is presented 50 times to the neural network and is classified 46 times correctly. It is miss-classified four times as ' 0 '. Character ' $b$ ' is classified 50 times correctly. Character ' $c$ ' is misclassified as ' $\mathrm{e}$ ' two times and one time each as 'o' and ' $u$ ' and is classified correctly 46 times. The average recognition accuracy of $88.76 \%$ is quiet good for this handwritten character recognition experiment using MLP as a classifier [3, 6, 7].

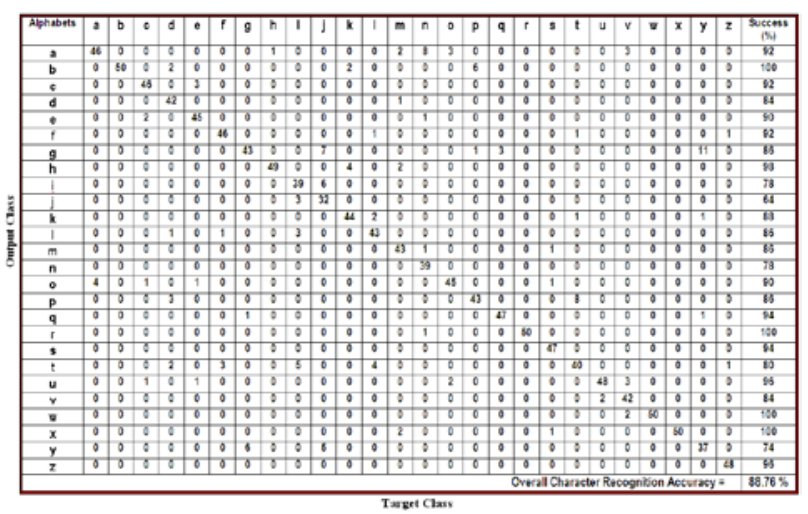

Figure 8. Confusion Matrix representing the MLP Classifier Performance using Hybrid Features. 


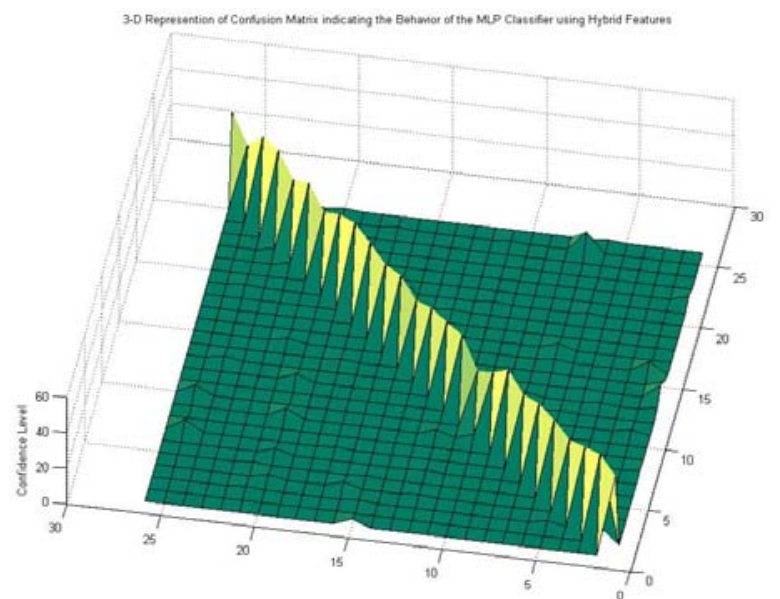

Figure 9. Three-Dimensional Plot of Confusion Matrix representing the MLP Behavior.

The RBF neural network has also been trained with 50 sets of each character i.e. $1300(50 \times 26=1300)$ character image samples from the database. Character ' $a$ ' is presented 50 times to the neural network and is classified 49 times correctly. It is miss-classified one times as 'o'. Character ' $\mathrm{j}$ ' is classified 42 times correctly and misidentified as character ' $i$ ' four times and two times each as character ' $g$ ' and ' $y$ ' out of a total of fifty trials. Recognition accuracy for each character (a-z) as well as overall recognition accuracy is displayed in Fig.10. The average recognition accuracy of $93.46 \%$ is excellent for this handwritten character recognition experiment using RBF as a classifier.

The recognition results obtained when MLP and RBF network classifiers are used to recognize the handwritten characters using Hybrid Features (features extracted from fusion of binarization and projection features) are shown in the form of confusion matrices as shown in Fig.8 and Fig.10 respectively. The confusion matrix shows the confusion among the recognized characters while testing the neural network's recognition accuracy $[6,7]$. The respective equivalent $3-D$ plots drawn in the 'MATLAB Environment' are also shown in Fig.9 and Fig. 11 respectively.

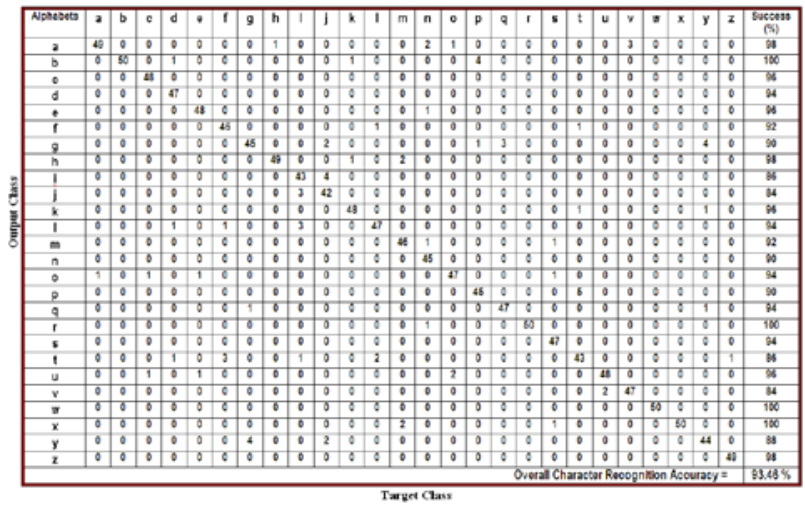

Figure 10. Confusion Matrix representing the RBF Network Performance using Hybrid Features.

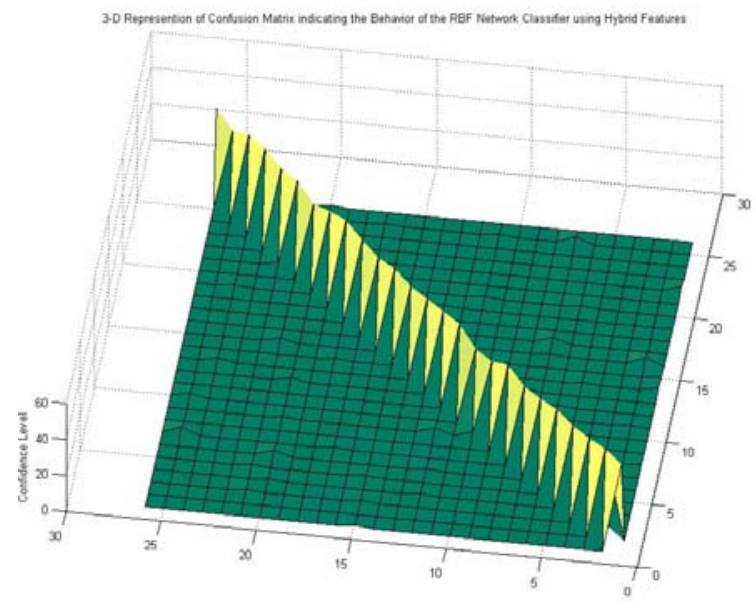

Figure 11. Three-Dimensional Plot of Confusion Matrix representing the RBF Network Behavior.

\section{CONCLUSION}

The recognition accuracy of a classifier system mainly depends on the quality of training samples, the techniques employed to extract features and the type of the classifier involved. The proposed method of fusion of character features obtained from binarization and projection profile techniques showed the remarkable enhancement in the performance for the handwritten character recognition using the MLP and RBF network classifiers. By using Hybrid features, the excellent character recognition accuracy of $88.76 \%$ and $93.46 \%$ is achieved for MLP and RBF classifier respectively.

\section{REFERENCES}

[1] S. N. Sivanandam, S. N. Deepa,” Principals of Soft Computing”, pp. 71-83. Wiley-India,

[2] A. Choudhary, R. Rishi, S. Ahlawat, "Unconstrained Handwritten Digit OCR Using Projection Profile and Neural Network Approach”. In: Proceedings of the InConINDIA 2012. AISC, vol. 132, pp. 119-126. Springer, Heidelberg, 2012.

[3] A. Choudhary, R. Rishi, "A Fused Feature extraction approach to OCR : MLP vs. RBF” in: ICT and Critical Infrastructure Proceedings of the 48th Annual Convention of CSI, CSI-2013 Vol.1 pp. 159-166, 2013

[4] A. Choudhary, R. Rishi, S. Ahlawat, "A New Character Segmentation Approach for Off-Line Cursive Handwritten Words”. Elsevier Procedia Computer Science 17, pp. 434-440, 2013.

[5] U. Bhattacharya, B. B. Chaudhuri, "A Majrity voting scheme for multiresolution recognition of handprinted numerals". in Seventh International Conference on Document Analysis and Recognition (ICDAR), vol. 1, p. 16, 2003.

[6] A. Choudhary, R. Rishi, "Improving the Character Recognition Efficiency of Feed Forward BP Neural Network”. International Journal of Computer Science and Information Technology (IJCSIT), vol. 3(1), pp. 85-96, 2011.

[7] A. Choudhary, R. Rishi, S. Ahlawat, "Handwritten Numeral Recognition Using Modified BP ANN Structure”. in: CCSIT 2011, Part III. CCIS, vol. 133, pp. 56-65, 2011.

[8] A. A. Desai, "Gujarati Handwritten Numeral Optical Character Recognition through Neural Network”. Pattern Recognition, vol. 43(7), pp. 2582-2589, 2010. 